\title{
Monensin Enhances the Binding of LDL to Human Hepatocyte-Like C3A Cells without Increasing the Number of LDL Receptors at the Plasma Membrane
}

\author{
Dayami Lopez ${ }^{1}$, DaTonye I Agina-Obu ${ }^{2}$, Catherine J Wooten ${ }^{3}$ \\ Department of Pharmaceutical Sciences, Biomanufacturing Research Institute and Technology Enterprise (BRITE) \\ College of Arts and Sciences, North Carolina Central University, Durham, NC 27707, USA
}

\begin{abstract}
The hepatic LDL receptor is the major determinant of plasma LDL levels, and as a result, a greater understanding of the regulatory mechanisms that control LDL receptor expression and function is essential. Herein, we optimized a biotinylation assay that was able to differentiate between cell surface (plasma membrane) and intracellular LDL receptors. We also tested monensin, a chemical that prevents the recycling of the LDL receptors to the cell surface and enhances the number of receptors that can bind and internalize LDL. Herein, it was confirmed the effects of monensin on the ability of the LDL receptor to bind LDL, and demonstrated for the first time, using the biotinylation assay, that monensin did not affect the LDL receptor expression levels at the plasma membrane or inside the cell. This was confirmed using immunocy to chemistry and Western blotting analysis. Monensin treatment may enhance the distribution of the LDL receptor to clathrin-coated pits explaining the higher binding of LDL, but not of VLDL, to the cells. This effect of monensin did not require upregulation of the LDL receptor expression. This indicates that the biotinylation assay described herein, in combination with monensin treatment, could be used in future studies to determine the effects of different treatments/drugs on the plasma membrane distribution/function of the hepatic LDL receptors.
\end{abstract}

Keywords: LDL, VLDL, LDL Receptor, Monensin, Hepatic Cells

\section{INTRODUCTION}

Atherosclerotic related diseases are still the leading causes of death and disability not only in the United States but also worldwide [1]. Increased levels and high oxidation of low density lipoprotein (LDL) particles are well-known risk factors for the development of atherosclerosis [2]. The primary function of the LDL receptor is the removal of the highly atherogenic LDL particles from the circulation before they become oxidized [3-5]. The LDL receptor pathway is responsible for removing $60-75 \%$ of all LDL particles in rats, $67 \%$ in rabbits, and up to $80 \%$ in humans [6-9]. The liver contains the higher expression (about 70\%) of LDL receptors compared to the rest of the body, so changes in plasma LDL levels are usually due to variations in the activity/expression of the hepatic LDL receptor [10].

The LDL receptor removes lipoproteins from the circulation through a pathway known as the cycling of the LDL receptor [11]. After a lipoprotein binds to the receptor at the cell surface, endocytosis of the lipoprotein/LDL receptor complex initiates by random nucleation of the clathrin-coated pits on the inner surface of the plasma membrane [11]. The LDL receptor adaptor protein-1 (LDLRAP1) has been shown to be important for efficient internalization of the hepatic LDL receptor within clathrin-coated pits [12, 13]. Further deformation of the membrane and polymerization of clathrin results in a coated vesicle attached to the plasma membrane by a narrow neck $[14,15]$. Dynamin, which is polymerized around the neck portion of the vesicle, hydrolyzes guanosine 5'triphosphate (GTP), and uses the energy released to drive the pinch off of the coated vesicle $[15,16]$. After clathrin is removed by uncoating enzymes, the vesicle is able to fuse with the endosome [17-19]. The activity of V-type ATPases present in the membrane of the endosome causes acidification of this organelle resulting in dissociation of the lipoprotein from the receptor [19-23].

At that point, the lipoprotein particle moves to the lysosome, where the cholesteryl ester portion is hydrolyzed to form cholesterol and free fatty acids, and the protein constituents are degraded into free amino acids [24, 25]. The majority of the LDL receptors are recycled back to the cell surface, where they can again bind and internalize lipoproteins $[26,27]$. In each round of the cycle, only a very small percentage of the LDL receptors are degraded

\footnotetext{
${ }^{1}$ Corresponding Author: lopezd@nccu.edu
} 
[17]. It is assumed that each LDL receptor molecule makes one complete circle every 10 minutes for a total of 150 trips in the 20-hours that the receptor last, before it is finally degraded [17]. Steady-state levels of the LDL receptor protein are maintained constant by replacing the degraded receptor with newly synthesized receptor molecules [17].

Monensin is a monovalent ion-selective ionophore that works mainly by exchanging sodium ions (in) for protons (out) across organelle membranes [28, 29]. As a result, any acidic intracellular compartment (i.e., trans-Golgi apparatus, lysosomes, and endosomes) is neutralized [28]. Among the cellular processes that are disrupted as a result of monensin treatment are 1) terminal glycosylation and proteolytic cleavages within the Golgi, 2) secretion of soluble proteins, and 3) movement of newly synthesized plasma-membrane proteins from the Golgi to the plasma membrane [28-31]. It is important to mention that independently of the inhibition by monensin, some incompletely processed proteins may still be secreted via unknown mechanisms that appear to bypass the Golgi apparatus [28]. During endocytosis of membrane bound receptors, such as the LDL receptor, monensin does not prevent their internalization, but it does prevent the intracellular release and degradation of internalized lipoproteins as well as the recycling of the internalized receptors [28]. Among the advantages of using monensin are 1) the low concentrations at which inhibition by monensin is achieved, 2) a minimum of side effects such as little or no change of protein synthesis or ATP levels, and 3) a reversible action [28]. In this study, we used monensin as a research tool to examine the lipoprotein binding ability of the LDL receptor in hepatic cells. We found that monensin enhanced the binding of LDL to human C3A cells without increasing the number of LDL receptors at the plasma membrane.

\section{MATERIALS AND METHODS}

\subsection{Materials}

The human hepatocyte-like C3A cell line was obtained from the American Type Culture Collection (Manassas, VA). Low glucose Dulbecco's modified Eagle's medium (LG-DMEM), penicillin/streptomycin solution, the Vybrant MTT cell proliferation assay kit, and fetal bovine serum (FBS) were purchased from Invitrogen (Carlsbad, $\mathrm{CA}$ ). $\mathrm{BD}^{\mathrm{TM}} \mathrm{MITO}+$ serum extender was from BD Biosciences (Sparks, MD). RIPA buffer, the BCA protein assay kit, Lane Marker Reducing Sample Buffer, precast 4-20\% SDS-PAGE, the SuperSignal West Pico chemiluminescence substrate, sulfo-NHS-SS-biotin, streptavidin magnetic beads, and the Fluorescence Biotin Quantitation kit were purchased from Pierce (Rockford, IL). Gentamicin solution, Ponceau S solution, HRP-labeled avidin, and monensin were obtained from Fisher Scientific (Pittsburg, PA). The rabbit anti-LDL receptor specific antibody used in most Western blotting studies (referred as "nAb") was kindly provided by Dr. Gene C. Ness (Department of Molecular Medicine, University of South Florida, Tampa, FL). Actin and all the HRP-labeled secondary antibodies were obtained from Santa Cruz Biotechnology (Santa Cruz, CA). The LDL Uptake CellBased Assay kit and the second rabbit anti-LDL receptor antibody used herein (referred as "cAb") were from Cayman Chemicals (Ann Arbor, MI). Dil (3,3'-dioctadecylindocarbocyanine)-VLDL was from Kalen Biomedical (Montgomery Village, MD). All other chemicals were purchased from Fisher Scientific or Sigma-Aldrich (St. Louis, MO).

\subsection{C3A Cell Culture}

Cells were maintained at a density of $10^{8}$ cells per $75-\mathrm{cm}^{3}$ flask in LG-DMEM medium supplemented with $10 \%$ FBS, penicillin (100 units $/ \mathrm{mL})$, streptomycin $(100 \mu \mathrm{g} / \mathrm{mL})$, and gentamicin $(30 \mu \mathrm{g} / \mathrm{mL})$ [maintaining medium] at $37^{\circ} \mathrm{C}$, under humidified atmosphere and $5 \%$ carbon dioxide. For the majority of the experiments, cells were set in $75-\mathrm{cm}^{3}$ flasks at the density of $2 \times 10^{6}$ viable cells per flask in maintaining medium. Twenty-four hours later, the medium was changed to a medium where the FBS has been replaced with $1 \mathrm{~mL} / \mathrm{L}$ of $\mathrm{BD}^{\mathrm{TM}} \mathrm{MITO}+$ serum extender (MITO+ medium). As previously reported, supplementing the medium with $\mathrm{BD}^{\mathrm{TM}} \mathrm{MITO}+$ serum extender does not cause detectable morphological and/or growth changes in C3A cells [32]. The MITO+ medium was used as a serum-free, lipid/cholesterol deficient medium [32]. For LDL binding and receptor expression studies using the cell-based assay from Cayman Chemicals, cells were set in black-opaque, clear bottom, 96-well plates at the density of $5 \times 10^{5}$ viable cells per well in maintaining medium. Once again, 24 hours later the medium was changed to MITO+ medium. The monensin concentration used to treat cells was $25 \mu \mathrm{M}$, which is the concentration previously recommended for this type of study [33]. After incubating in MITO+ medium for 24 hours, monensin was added, and cells were incubated for $0,15,30,45$, and 60 minutes. Cells were processed as indicated below for each specific experiment.

\subsection{Preparation of RIPA Proteins and Western Blotting Analysis}

Lysates (referred as "RIPA proteins") were prepared using ice-cold RIPA buffer (25 mM Tris-HCl, pH 7.6, $150 \mathrm{mM}$ $\mathrm{NaCl}, 1 \% \mathrm{NP}-40,1 \%$ sodium deoxycholate, $0.1 \%$ SDS, and protease and phosphate inhibitors) according to the 
manufacturer's recommendations. Protein concentrations were determined using the BCA protein assay. Equivalent amounts of proteins were denatured in reducing sample buffer at $70^{\circ} \mathrm{C}$ for 5 minutes and subjected to electrophoresis on precast 4-20\% SDS-PAGEs. Electroblotting onto nitrocellulose membranes, staining with $0.1 \%$ Ponceau S (in 5\% acetic acid) to verify protein loading, and Western blotting analysis were performed using standard methods. Primary antibodies used herein were rabbit anti-LDL receptor nAb (diluted 1:2000) or $\mathrm{cAb}$ (diluted 1:1000), and goat anti-actin antibody (diluted 1:250; internal control). The blocking agent was 5\% non-fat dry milk-TTBS (non-biotinylated proteins and proteins eluted from the magnetic beads) or 3\% BSA-TBS (biotinylated proteins). Primary antibodies bound to membranes were detected using HRP-labeled secondary antibodies and the SuperSignal West Pico chemiluminescence substrate. Biotinylated proteins were also detected with avidin-HRP (1:1000 dilution). Multiple exposures ranging from 0.5 seconds to 20 minutes and quantitation of the exposures were made using a Kodak Image Station 4000R Pro Imaging System and the Kodak Molecular Imaging software (New Haven, CT).

\subsection{LDL Uptake/Immunocy to chemistry for LDL Receptor Expression Using a Cell-Based Assay}

This assay was carried out using the LDL Uptake Cell-Based Assay Kit (Cayman Chemicals). Briefly, cells were plated in black opaque plates and treated with and without monensin in MITO+ medium as indicated above. After the treatment, the medium was replaced with ice-cold MITO+ medium containing $10 \mu \mathrm{g} / \mathrm{mL}$ of LDL-DyLight 549 . Cells were incubated for 1 hour at $4^{\circ} \mathrm{C}$ to allow LDL binding. The medium was then aspirated, and the cells were washed twice with ice-cold PBS. After the second wash, fresh ice-cold PBS was added to the cells. LDL binding data were collected using a BMG Labtech PHERAstar ${ }^{\mathrm{TM}}$ fluorescent plate reader $(\mathrm{ex} / \mathrm{em}=540 / 570 \mathrm{~nm}$ ). Immunocytochemistry to determine LDL receptor protein expression was then carried out. First, the PBS was removed followed by washing once with TBS, $\mathrm{pH}$ 7.4. Fixation, washing with TBST three times, and blocking were carried out according to the kit's instructions. Incubation with the rabbit anti-LDL receptor $\mathrm{cAb}$ (provided in the kit) was performed overnight at $4^{\circ} \mathrm{C}$. After washing three times with TBST, the cells were incubated for 1 hour with the DyLight 488-conjugated anti-rabbit antibody. Once again, cells were washed with TBST. LDL receptor expression data were collected using a BMG Labtech PHERAstar ${ }^{\mathrm{TM}}$ fluorescent plate reader $(\mathrm{ex} / \mathrm{em}=485 / 535 \mathrm{~nm}$ ). The fluorescent signal obtained for each condition/experiment was corrected by subtracting the fluorescence of unlabeled cells (background autofluorescence) in each experiment.

\subsection{Tetrazolium-Based Colorimetric Assay (MTT)}

To determine whether monensin affects the viability of the C3A cells, cell viability studies were carried using the Vybrant MTT cell proliferation assay. For this, $1 \times 10^{4} \mathrm{C} 3 \mathrm{~A}$ cells were plated per well onto clear 96-well plates in maintaining medium. Treatment with $25 \mu \mathrm{M}$ monensin for $0,15,30,45$, and 60 minutes in MITO+ medium was carried out as described above. After the treatment, cells were exposed to the MTT [3-(4,5-dimethylthiazol-2-yl)2,5-diphenyltetrazolium bromide] dye $(5 \mathrm{mg} / \mathrm{mL})$ and incubated at $37^{\circ} \mathrm{C}$ for 2 hours. The resulting formazan crystals were solubilized with DMSO, and the absorbance was measured at $540 \mathrm{~nm}$ with a BMG Labtech PHERAstar ${ }^{\mathrm{TM}}$ plate reader.

\subsection{DII-VLDL Internalization Studies}

For this experiment, cells were plated in black opaque/clear bottom 96 well plate. After treating with monensin in MITO+ medium for 45 minutes as described above, the medium was replaced with ice-cold medium containing 1.0 $\mu \mathrm{g} / \mathrm{ml}$ of DiI-VLDL. DiI-VLDL binding was allowed by incubating for 1 hour at $4^{\circ} \mathrm{C}$. Binding data were collected using a BMG Labtech PHERAstar ${ }^{\mathrm{TM}}$ fluorescent plate reader (ex/em: 554/571 nm). Once again, the fluorescent signal obtained for each condition/experiment was corrected by subtracting the fluorescence of unlabeled cells (background autofluorescence) in each experiment.

\subsection{Cell Surface Biotinylation Studies}

After treating with and without monensin in MITO+ medium, cells were cooled on ice for 30 minutes to stop receptor cycling followed by three washes with ice-cold PBS. Biotinylation of plasma membrane proteins was carried by incubating cells for 2 hours at $4^{\circ} \mathrm{C}$ with $0.8 \mathrm{mM}$ of the membrane impermeable sulfo-NHS-SS-biotin (Sulfosuccinimidyl-2-(biotinamido)-ethyl-1,3'-dithiopropionate) dissolved in ice-cold PBS. At that point, cells were washed once with $100 \mathrm{mM}$ Tris-HCl, $\mathrm{pH}$ 7.7, followed by incubation in a fresh aliquot of $100 \mathrm{mM}$ Tris-HCl, $\mathrm{pH}$ 7.7, for 30 minutes at $4^{\circ} \mathrm{C}$, to quench any residual biotin reagent. After washing twice in ice-cold PBS, cell extracts were prepared in immunoprecipitation buffer $(0.25 \mathrm{M}$ sucrose, $0.05 \%$ SDS, $1 \%$ triton X-100, $0.5 \%$ sodium deoxycholate, $0.32 \mathrm{M} \mathrm{NaCl}, 2 \mathrm{mM} \mathrm{CaCl}_{2}$, and protease inhibitors). Protein concentrations were measured using the $\mathrm{BCA}$ assay. The incorporation of biotin into plasma membrane proteins was confirmed using the Fluorescence Biotin 
Quantitation kit from Pierce ( $\mathrm{p}=0.884 ; \mathrm{n}=6$; data not shown) or Western blotting analysis using avidin-HRP for detection. Four hundred $\mu \mathrm{g}$ of cell lysates were incubated overnight at $4^{\circ} \mathrm{C}$ with $100 \mu \mathrm{L}$ of $50 \%$ streptavidinmagnetic beads in $1.5 \mathrm{~mL}$ of dilution buffer $(1 \mathrm{mg} / \mathrm{mL}$ BSA, $0.1 \%$ triton X-100, $1 \mathrm{mM}$ Tris- $\mathrm{HCl}, \mathrm{pH} 8.0,140 \mathrm{mM}$ $\mathrm{NaCl}$, and protease inhibitors). Separation reactions were carried out by incubating the lysates with streptavidin magnetic beads overnight at $4^{\circ} \mathrm{C}$ with rotation. A magnetic stand was used to separated the supernatant (containing non-biotinylated intracellular proteins) from the magnetic beads (containing the biotinylated plasma membrane proteins). Non-biotinylated proteins were concentrated using 4 volumes of methanol and then separated using electrophoresis. The biotinylated proteins were eluted from the magnetic beads by first resuspending the beads in $1 \mathrm{X}$ reducing sample buffer supplemented with $50 \mathrm{mM}$ DTT and then heating the mixture at $70^{\circ} \mathrm{C}$ for 5 minutes. After separation using the magnetic stand, the supernatants (containing eluted plasma membrane proteins - no longer biotinylated) were removed and separated using electrophoresis. Western blotting analysis for the LDL receptor was performed as described above.

\subsection{Statistical Analysis}

Data from the individual parameters were compared by analysis of variance (ANOVA) followed by StudentNewman-Keuls multiple comparison test or unpaired t-test, when applicable, using the GraphPad Prism 6 software (GraphPad Software, Inc., La Jolla, CA). A p <0.05 was considered significant for all tests.

\section{RESULTS}

We started by examining the effects of monensin on the LDL receptor protein expression in the human hepatocytelike C3A cell line. For this, cells were treated with and without monensin $(25 \mu \mathrm{M})$ for 45 minutes in MITO+ medium. After the incubation, RIPA proteins were prepared and analyzed by Western blotting. Action was used as the internal control for these studies. Fig. 1A illustrates representative Western blots for LDL receptor and action.

A

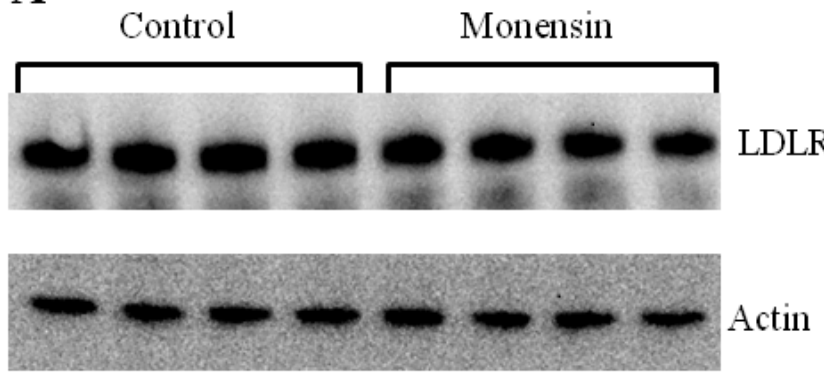

B

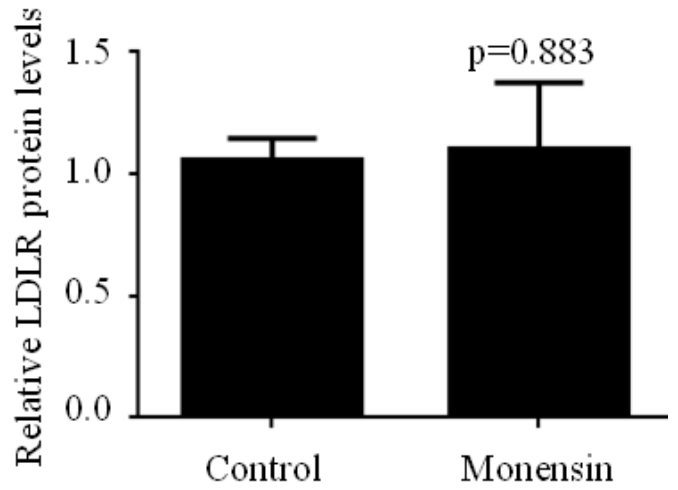

C

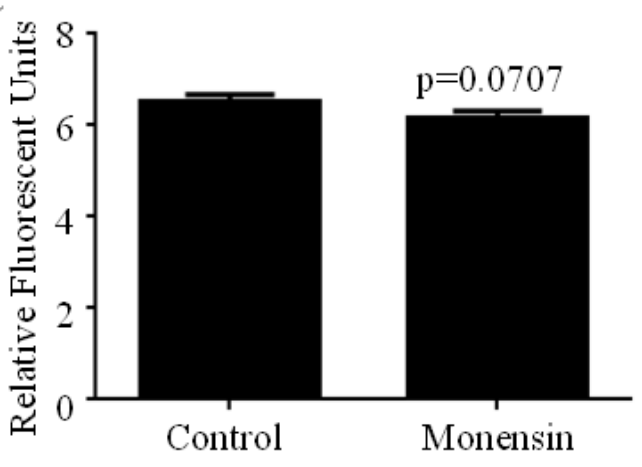

Fig. 1 Effects of monensin on LDL receptor protein expression in human hepatocyte-like C3A cells 
Treatment with $25 \mu \mathrm{M}$ of monensin was carried out for 45 minutes. (A) Typical Western blots for human LDL receptor (LDLR) and actin (internal control) are shown. Twenty $\mu \mathrm{g}$ of total protein was loaded per lane. (B) Quantitated results for three independent Western blot experiments. Data are presented as relative LDL receptor protein level mean (corrected using the actin for the same sample) \pm standard error of the mean (SEM) for $n=9$. (C) Immunocytochemistry results obtained using a cell-based assay. The fluorescent signal corresponding to the expression levels of LDL receptor protein (background subtracted) were measured using a BMG Labtech PHERAstar ${ }^{\mathrm{TM}}$ fluorescent plate reader $(\mathrm{ex} / \mathrm{em}=485 / 535 \mathrm{~nm})$. Data are presented as relative fluorescent unit mean \pm SEM for $n=40$ corresponding to three independent experiments. "p" values in (B) and (C) were obtained by comparing control and monensin treated groups with the unpaired t-test using the GraphPad Prism 6 software.

As shown in Fig. 1B, no significant differences $(p=0.883 ; n=9)$ in protein expression levels for the LDL

receptorwere observed when comparing control and monensin treated samples. Similar results were obtained when incubating with monensin for 15 and 30 minutes ( $\mathrm{p}=0.6146$ obtained using ANOVA; $\mathrm{n}=3$ per treatment group; data not shown). Incubating with monensin beyond 45 minutes caused that the LDL receptor and actin signals appeared in the Western blots more as smears than single bands. A possible explanation for these findings could be that monensin partially inhibited glycosylation/protein processing within the Golgi apparatus [28-31] resulting in the appearance of immunoreactive proteins of different molecular weights. We did not see any statistically significant difference in the levels of glycosylated ( $\mathrm{p}=0.2447$ for $\mathrm{nAb} ; \mathrm{p}=0.8169$ for $\mathrm{cAb}$ ) and unglycosylated $(\mathrm{p}=0.1360$ for $\mathrm{nAb} ; \mathrm{p}=0.5738$ for $\mathrm{cAb}$ ) isoforms of the LDL receptor, when comparing control and monensin treated samples, as long as the treatment was carried out for 45 minutes or less ( $\mathrm{n}=3$; data not shown).

To confirm the Western blot results, immunocytochemistry to detect the LDL receptor was performed using a cellbased assay. The fluorescence signal obtained from incubating with the anti-LDL receptor and the Dylight 488labeled anti-rabbit antibodies was at least 4 times higher $(p<0.001 ; n=40)$ than the background fluorescence from unlabeled cells (data not shown). As shown in Fig. 1C, monensin treatment had no significant effect on the expression levels of the LDL receptor protein in C3A cells ( $\mathrm{p}=0.0707 ; \mathrm{n}=40$ ).

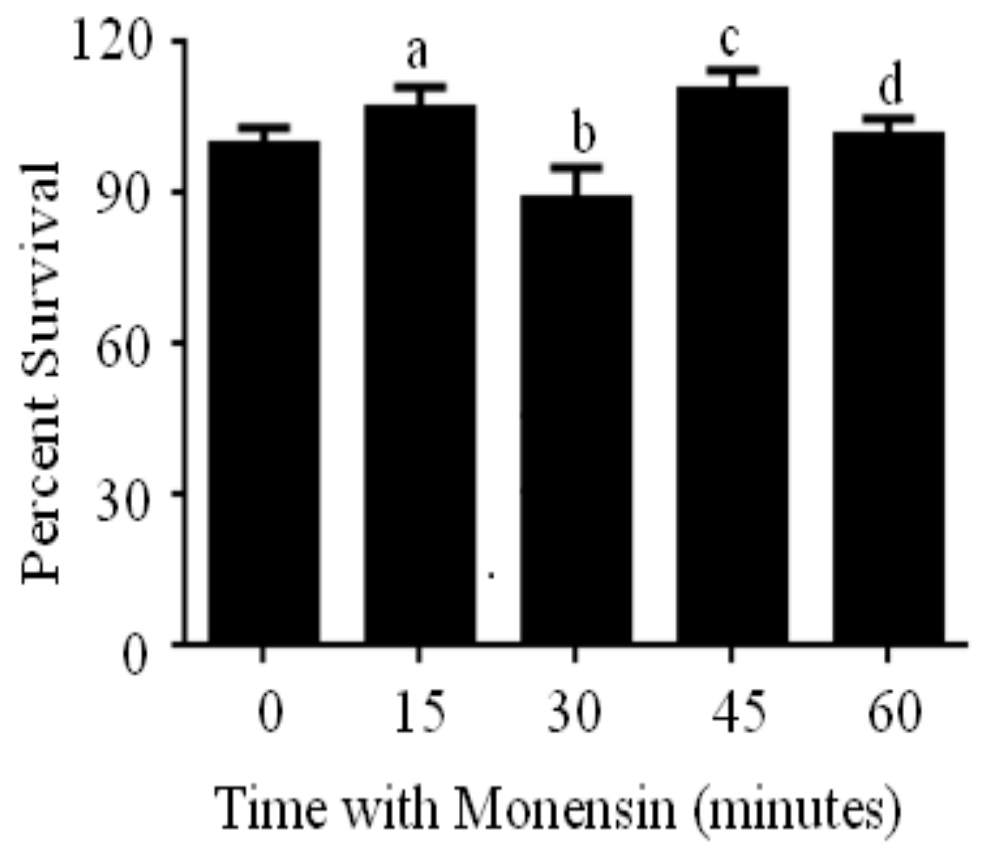

Fig. 2 Effects of monensin on the viability of C3A cells

Treatment with $25 \mu \mathrm{M}$ of monensin was carried out for the indicated times. Typical data collected using the MTT assay are presented as mean percent survival \pm SEM for $n=12$ per treatment group. "p" values were obtained by comparing control ("0" time point) and monensin treated ("15" to "60" time points) groups with analysis of variance (ANOVA) followed by Student-Newman-Keuls multiple comparison test using the GraphPad Prism 6 software. "a" refers to $\mathrm{p}=0.1987$; " $\mathrm{b}$ " refers to $\mathrm{p}=0.1369$; "c" refers to $\mathrm{p}=0.0694$, and "d" refers to $\mathrm{p}=0.6975$. 
Because of the smearing in the protein signals seen in the Western blot for both LDL receptor and actin, when incubating with monensin for more than 45 minutes, the next step was to carry out MTT assays to determine whether monensin was able to affect the viability of the C3A cells. For this experiment, cells were plated in clear 96 well plates and treated with $25 \mu \mathrm{M}$ of monensin from 0 (referred to as control group) up to 60 minutes (monensin treated groups) in MITO+ medium. The results for the MTT assays are shown in Fig. 2. As illustrated, there were not significant differences $(n=12)$ in the viability of the cells incubated with monensin, even at 45 and 60 minutes, as compared to the control sample ("0" time point; Fig. 2). These results indicated that the smearing of the LDL receptor and actin signals seen in the Western blots were not due to changes in the viability of the cells upon monensin treatment.
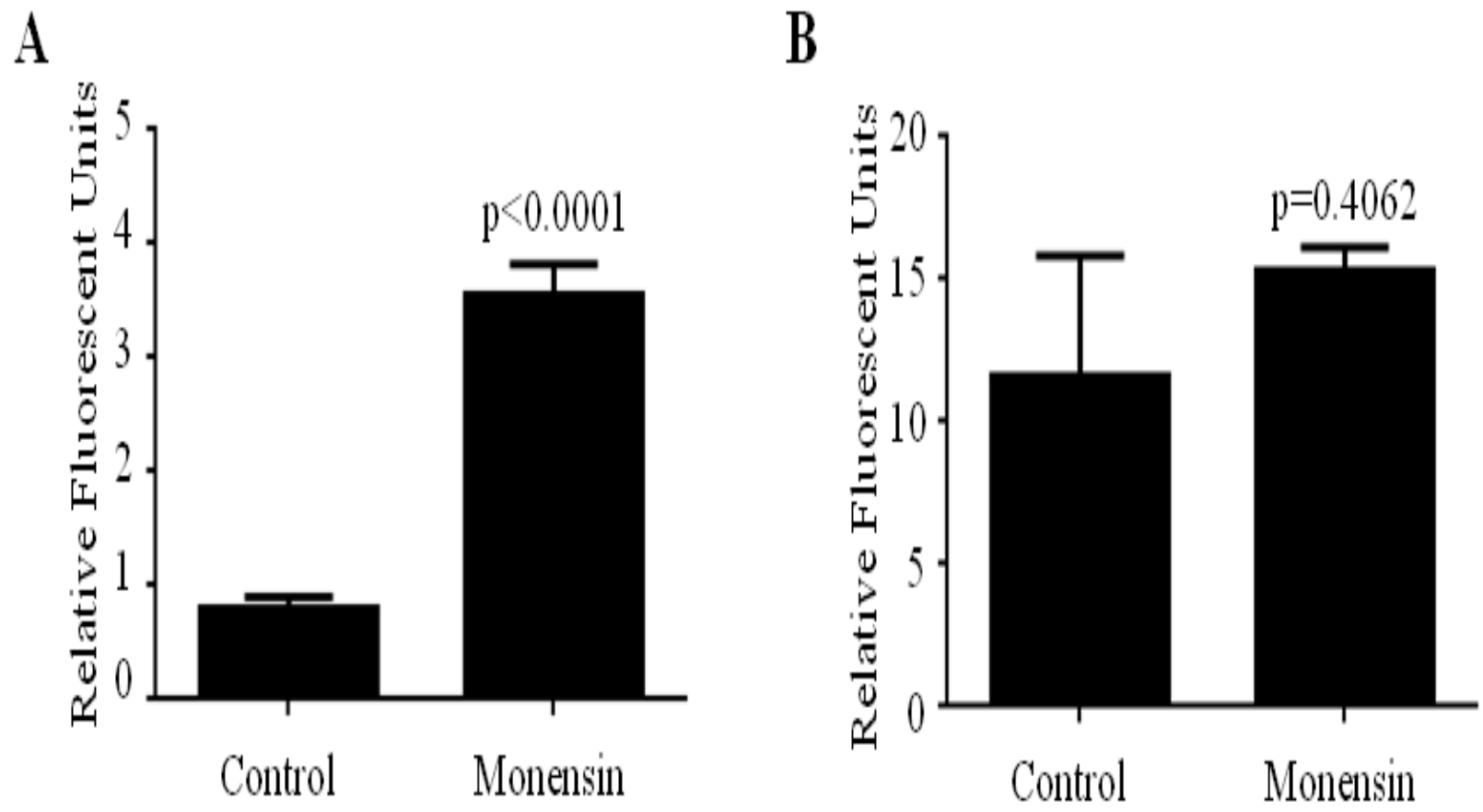

Fig. 3 Effects of monensin on the binding of lipoproteins to C3A cells

Treatment with monensin for 45 minutes was done as described above. Labeling with DyLight 549-LDL (A) or DiIVLDL (B) was analyzed using a BMG Labtech PHERAstar ${ }^{\mathrm{TM}}$ fluorescent plate reader. Data are expressed as relative fluorescent unit mean \pm SEM ( $n=12$ for DyLight 549-LDL; $n=8$ for DiI-VLDL). "p" values obtained by comparing control and monensin treated groups as described in Fig. 1.

To determine whether this increase in LDL binding to the C3A cells in response to monensin treatment was not due to an increase in the number of LDL receptors present at the plasma membrane, biotinylation studies were carried out.

At that point, we wanted to investigate whether monensin could affect the binding of lipoprotein particles to the C3A cells. For this, cells were plated in black opaque 96 well plates and treated with and without monensin as described above. DyLight 549-LDL binding at $4^{\circ} \mathrm{C}$ was carried out for 1 hour. Cells labeled with DyLight 549LDL were detected using a BMG Labtech PHERAstar fluorescent plate reader. The fluorescence signal obtained from incubating with DyLight 549-LDL was at least 1.453 times higher $(\mathrm{p}<0.0001 ; n=12)$ than the background fluorescence from unlabeled cells (data not shown). As depicted in Fig. 3A, monensin treatment resulted in a significant induction (4.44-fold; $\mathrm{n}=12 ; \mathrm{p}<0.0001$ ) in the binding of LDL to the C3A cells. We also performed binding studies with BODIPY ${ }^{\circledR}-$ LDL followed by detection using flow cytometry as previously described [34] and obtained comparable results (5.33-fold induction by monensin treatment; $\mathrm{p}=0.0026 ; \mathrm{n}=8$; data not shown). 
Interestingly, the binding of DiI-VLDL to the C3A cells was only slightly increased (1.31-fold) by the presence of monensin, but this increase was not statistically significant ( $\mathrm{p}=0.4062$; Fig. 3B).

A

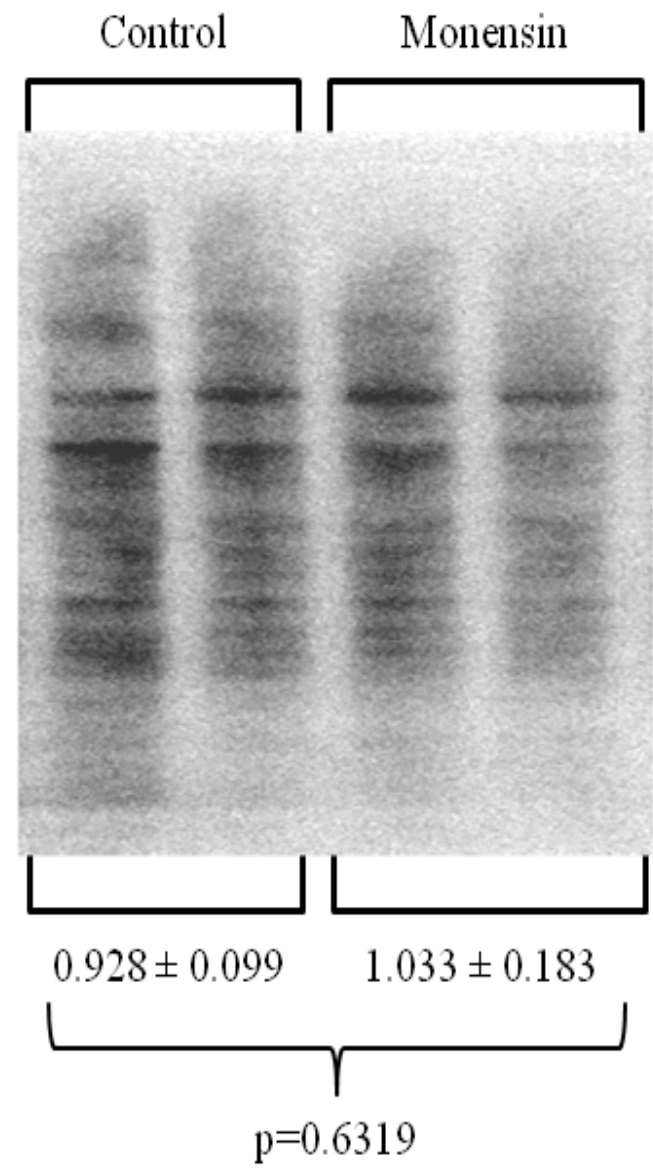

B

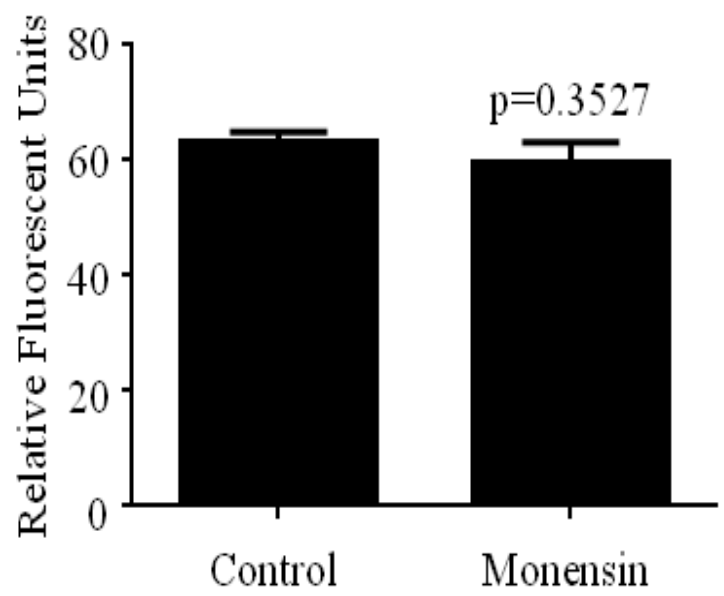

C

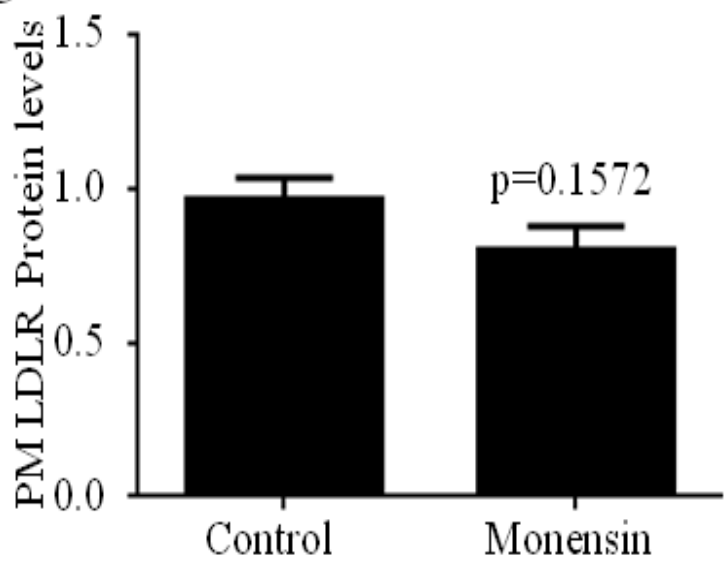

Fig. 4 Effects of monensin on plasma membrane expression of the LDL receptor.

Treatment with monensin was carried out as indicated above. Biotinylation of plasma membrane proteins followed by separation of biotinylated and non-biotinylated proteins using streptavidin-magnetic beads were performed. (A) Determination of the biotin incorporation using avidin-HRP. A typical Western blot for total biotinylated proteins detected using avidin-HRP is shown. Quantitated results (mean $\pm \mathrm{SEM}$; for $\mathrm{n}=4$ per treatment condition) are shown below the blot. (B) Determination of biotin incorporation using a fluorescence biotin quantitation assay. Data are expressed as relative fluorescent unit mean \pm SEM for $n=6$ per treatment condition. (C) Western blotting results for plasma membrane LDL receptor (PM LDLR). Data are presented as relative LDL receptor (LDLR) protein level mean \pm SEM for $n=4$ per treatment condition. " $p$ " values were obtained by comparing control and monensin treated groups as described above

As shown in Fig. 4A ( $\mathrm{p}=0.6319$ ) and Fig. 4B ( $\mathrm{p}=0.3527$ ), there was no significant difference in the amount of biotin that was incorporated into the cellular proteins. The biotinylated (plasma membrane; PM) proteins were separated from the non-biotinylated (intracellular) proteins using streptavidin magnetic beads. Western blotting analysis was 
then carried out to detect the LDL receptor. As shown in Fig. 4C, the amount of LDL receptor protein present at the plasma membrane (PM LDLR) was unaffected by treating with monensin $(\mathrm{p}=0.1572)$. Similarly, we didn't see any significant differences in the amount of intracellular LDL receptor protein present in control and monensin treated cells (data not shown; $\mathrm{p}=0.2447 ; \mathrm{n}=3$ per treatment condition). These data suggested that the increase in LDL binding as a result of monensin treatment was not due to an increased number of LDL receptors at the plasma membrane.

\section{DISCUSSION}

It has been previously shown that independently of whether the function of the LDL receptor is enhanced or reduced, the overall expression levels of the LDL receptor protein remain unchanged [35]. Under these conditions, the LDL receptor mRNA levels are changed in a direct correlation with changes in receptor function [35]. In that study, the expression of the LDL receptor protein was usually measured in total membrane preparations or total cellular extracts without differentiation between cell surface and intracellular receptors [35]. Thus, a question deriving from those findings was whether the treatments were actually able to affect the expression of the LDL receptor at the cell surface in direct correlation with their effects on receptor function.

In the study described herein, we used a biotinylation assay that specifically labeled plasma membrane proteins, fluorescent lipoprotein binding assays, and immunocytochemistry studies to examine the specific cellular localization and the lipoprotein binding ability (indicator of function) of the LDL receptor in C3A cells treated with and without monensin. Monensin is a chemical that has been used extensively to examine the localization and function of the LDL receptors in different cell lines [33, 36-38]. This chemical not only prevents the recycling of the LDL receptor to the cell surface, but also enhances the number of receptors that can bind and internalize LDL [33, 36-38]. In our study, we were able to confirm the effects of monensin on the ability of the LDL receptor to bind LDL, but also demonstrated, using a biotinylation assay, that monensin did not affect the LDL receptor expression levels at the plasma membrane or inside the cell. This was confirmed using immunocytochemistry and Western blotting analysis.

According to several studies, the LDL receptor could internalize different lipoprotein particles via diverse pathways [39-41]. The efficient internalization of LDL particles, for example, has been shown to require the acid-dependent mechanism of lipoprotein release in the endosome and the presence of an intact LDL receptor adaptor protein-1 (LDLRAP1), factors that are not required for the internalization of VLDL and/or $\beta$-VLDL [39, 40]. This suggests that the removal of LDL by the receptor needs an intact clathrin-dependent internalization pathway, whereas internalization of VLDL can occur through an alternative pathway like caveolae. In fact, it has been shown that mice lacking caveolin-1 have elevated levels of VLDL but not of LDL supporting this hypothesis [41].

We have previously demonstrated that C3A cells grown in MITO+ medium have more LDL receptors in the clathrin-coated pits than in caveolae and that treating rats with zaragozic acids (ZA), a potent cholesterol biosynthesis inhibitor, increases the distribution of the LDL receptor towards the clathrin-coated pits [34]. Thus, it might be possible that monensin treatment enhanced the distribution of the LDL receptor to clathrin-coated pits explaining the higher binding of LDL, but not of VLDL, to the cells. As expected due to the short time used for the incubation with monensin, no changes in LDL receptor mRNA expression were observed in our studies (data not shown). Thus, it was very likely that the effects of monensin on the binding of LDL to the C3A cells seen in our studies were due to redistribution of the LDL receptor within the plasma membrane and not to an overall change in the LDL receptor expression. Further studies are required to confirm the distribution of the LDL receptor within plasma membrane microdomains in response to monensin treatment. This indicates that the biotinylation assay described herein in combination with monensin treatment could be used in future studies to determine the effects of different treatments/drugs on the microdomain distribution of the plasma membrane LDL receptors and the relationship of this distribution with the receptor function.

\section{ACKNOWLEDGEMENTS}

The project described was supported by funds from the State of North Carolina. The authors would like to acknowledge the support of the Golden LEAF Foundation, BRITE, and Drs. Li-An Yeh and David S. Thomson. We thank Dr. Gene C. Ness (Department of Molecular Medicine, University of South Florida, Tampa, FL) for kindly providing the rabbit anti-LDL receptor antibody.

\section{REFERENCES}


[1] C.J. Murray, A.D. Lopez (1997) Mortality by cause for eight regions of the world: Global Burden of Disease Study. Lancet 349:1269-1276.

[2] J.A. Berliner, M. Navab, A.M. Fogelman, et al. (1995) Atherosclerosis: Basic Mechanisms Oxidation, Inflammation, and Genetics. Circulation 91:2488-2496.

[3] R.W. Bishop (1992) Structure of the hamster low density lipoprotein receptor gene. J Lipid Res 33:549-557.

[4] M.J.V. Hoffer, M.M. van Eck, F. Petris, et al. (1993) The mouse low density lipoprotein receptor gene: cDNA sequence and exon-intron structure. Biochem Biophys Res Commun 191:880-886.

[5] M.H. Hofker, R.R. Frants (1993) The mouse low density lipoprotein receptor gene: cDNA sequence and exonintron structure. Biochem Biophys Res Commun 191:880-886.

[6] H.R. Koelz, B.C. Sherrill, S.D. Turley, J.M. Dietschy (1982) Correlation of LDL and HDL binding in vivo with rates of lipoprotein degradation in the rat. A comparison of lipoproteins of rat and human origin. J Biol Chem 257:8061-8072.

[7] J.M. Dietschy, D.K. Spady, J.B. Meddings (1988) A quantitative approach to low density lipoprotein metabolism in man and in various experimental animals. In Hyperlipidaemia and Atheroscerosis. Editors: Suckling KE \& Groot PHE; Academic Press, London, pp. 25-39.

[8] D.W. Billheimer, Y. Watanabe, T. Kita (1982) Impaired receptor-mediated catabolism of low density lipoprotein in the WHHL rabbit, an animal model of familial hypercholesterolemia. Proc Natl Acad Sci USA 79:3305-3309.

[9] D.W. Billheimer, J.S. Stone, S.M. Grundy (1979) Metabolic studies in familial hypercholesterolemia. Evidence for a gene-dosage effect in vivo. J Clin Invest 64:524-533.

[10] D.W. Billheimer, J.L. Goldstein, S.C. Grundy, et al. (1984) Liver transplantation to provide LDL receptors and lower plasma cholesterol in a child with homozygous familiar hypercholesterolemia. N Engl J Med 311:16581664.

[11] J.L. Goldstein, S.K. Basu, M.S. Brown (1983) Receptor-mediated endocytosis of low-density lipoprotein in cultured cells. Methods in Enzymol 98:241-260.

[12] G. He, S. Gupta, M. Yi, et al. (2002) ARH is a modular adaptor protein that interacts with the LDL receptor, clathrin and AP-2. J Biol Chem 277:44044-44049.

[13] S.K. Mishra, S.C. Watkins, L.M. Traub (2002) The autosomal recessive hypercholesterolemia (ARH) protein interfaces directly with the clathrin-coat machinery. Proc Natl Acad Sci USA 99:16099-16104.

[14] P.D. Blood, G.A. Voth (2006) Direct observation of Bin/amphiphysin/Rvs (BAR) domain-induced membrane curvature by means of molecular dynamics simulations. Proc Natl Acad Sci USA 103:15068-15072.

[15] Y. Yoshida, M. Kinuta, T. Abe, et al. (2004) The stimulatory action of amphiphysin on dynamin function is dependent on lipid bilayer curvature. EMBO J 23:3483-3491.

[16] S. Sever, J. Skoch, S. Newmyer, et al. (2006) Physical and functional connection between auxilin and dynamin during endocytosis. EMBO J 25:4163-4174.

[17] M.S. Brown, R.G. Anderson, J.L. Goldstein (1983) Recycling receptors: the round-trip itinerary of migrant membrane proteins. Cell 32:663-667.

[18] P. Ghosh, S. Kornfeld (2003) AP-1 binding to sorting signals and release from clathrin-coated vesicles is regulated by phosphorylation. J Cell Biol 160:699-708.

[19] X. Arias-Moreno, A. Velazquez-Campoy, J.C. Rodríguez, et al. (2008) Mechanism of low density lipoprotein (LDL) release in the endosome: implications of the stability and $\mathrm{Ca} 2+$ affinity of the fifth binding module of the LDL receptor. J Biol Chem 283:22670-22679.

[20] N. Beglova, H. Jeon, C. Fisher, S.C. Blacklow (2004) Cooperation between fixed and low pH-inducible interfaces controls lipoprotein release by the LDL receptor. Mol Cell 16:281-292.

[21] T. Yamamoto, H.C. Chen, E. Guigard, et al. (2008) Molecular studies of pH-dependent ligand interactions with the low-density lipoprotein receptor. Biochemistry 47:11647-11652.

[22] N. Beglova, S.C. Blacklow (2005) The LDL receptor: how acid pulls the trigger. Trends Biochem Sci 30:309317.

[23] S. Huang, L. Henry, Y.K. Ho, et al. (2009) Mechanism of LDL Binding and Release Probed By StructureBased Mutagenesis of the LDL Receptor. J Lipid Res 51:297-308. 
[24] J. Gent, I. Braakman (2004) Low-density lipoprotein receptor structure and folding. Cell Mol Life Sci 61:2461-2470.

[25] G. Rudenko, L. Henry, K. Henderson, et al. (2002) Structure of the LDL receptor extracellular domain at endosomal pH. Science 298:2353-2358.

[26] H. Jeon, W. Meng, J. Takagi, et al. (2001) Implications for familial hypercholesterolemia from the structure of the LDL receptor YWTD-EGF domain pair. Nat Struct Biol 8:499-504.

[27] N. Beglova, H. Jeon, C. Fisher, S.C. Blacklow (2004) Structural features of the low-density lipoprotein receptor facilitating ligand binding and release. Biochem Soc Trans 32:721-723.

[28] H.H. Mollenhauer, D.J. Morré, L.D. Rowe (1990) Alteration of intracellular traffic by monensin; mechanism, specificity and relationship to toxicity. Biochim Biophys Acta 1031:225-246.

[29] A. Tartakoff, P. Vassalli, M. Détraz (1978) Comparative studies of intracellular transport of secretory proteins. J Cell Biol 79:694-707.

[30] J.C. Johnson, M.J. Schlesinger (1980) Vesicular stomatitis virus and sindbis virus glycoprotein transport to the cell surface is inhibited by ionophores. Virology 103:407-424.

[31] G.J. Strous, H.F. Lodish (1980) Intracellular transport of secretory and membrane proteins in hepatoma cells infected by vesicular stomatitis virus. Cell 22:709-717.

[32] C.J. Wooten, A.F. Adcock, D.I. Agina-Obu, D. Lopez (2014) Having excess levels of PCSK9 is not sufficient to induce complex formation between PCSK9 and the LDL receptor. Arch Biochem Biophys 545C:124-132.

[33] S.K. Basu, J.L. Goldstein, R.G. Anderson, M.S. Brown (1981) Monensin interrupts the recycling of low density lipoprotein receptors in human fibroblasts. Cell 24:493-502.

[34] S. Ivaturi, C.J. Wooten, M.D. Nguyen, et al. (2014) Distribution of the LDL receptor within clathrin-coated pits and caveolae in rat and human liver. Biochem Biophys Res Commun 445:422-427.

[35] D. Lopez, C.M. Chambers, R.K. Keller, G.C. Ness (1998) Compensatory responses to inhibition of hepatic squalene synthase. Arch Biochem Biophys 351:159-166.

[36] R.G. Anderson, J.L. Goldstein, M.S. Brown (1976) Localization of low density lipoprotein receptors on plasma membrane of normal human fibroblasts and their absence in cells from a familial hypercholesterolemia homozygote. Proc Natl Acad Sci USA 73:2434-2438.

[37] R.G. Anderson, J.L. Goldstein, M.S. Brown (1977) A mutation that impairs the ability of lipoprotein receptors to localize in coated pits on the cell surface of human fibroblasts. Nature 270:695-699.

[38] B.D. Grant, M. Sato (2006) Intracellular trafficking. Worm Book 21:1-9.

[39] Z. Zhao, P. Michaely (2009) The role of calcium in lipoprotein release by the low-density lipoprotein receptor. Biochemistry 48:7313-7324.

[40] C. Jones, R. Garuti, P. Michaely, et al. (2007) Disruption of LDL but not VLDL clearance in autosomal recessive hypercholesterolemia. J Clin Invest 117:165-174.

[41] B. Razani, T.P. Combs, X.B. Wang, et al. (2002) Caveolin-1-deficient mice are lean, resistant to diet-induced obesity, and show hypertriglyceridemia with adipocyte abnormalities. J Biol Chem 277:8635-8647. 